\title{
Testing Logselfsimilarity of Soil Particle Size Distribution: Simulation with Minimum Inputs
}

\author{
Carlos García-Gutiérrez, and Miguel Ángel Martín
}

\begin{abstract}
Particle size distribution (PSD) greatly influences other soil physical properties. A detailed textural analysis is time-consuming and expensive. Soil texture is commonly reported in terms of mass percentages of a small number of size fractions (typically, clay, silt and sand). A method to simulate the PSD from such a poor description or even from the poorest description, consisting in the mass percentages of only two soil size fractions, would be extremly useful for prediction purposes. The goal of this paper is to simulate soil PSDs from the minimum number of inputs, i.e., two and three textural fraction contents, by using a logselfsimilar model and an iterated function system constructed with these data. High quality data on 171 soils are used. Additionally, the characterization of soil texture by entropy-based parameters provided by the model is tested. Results indicate that the logselfsimilar model may be a useful tool to simulate PSD for the construction of pedotransfer functions related to other soil properties when textural information is limited to moderate textural data.
\end{abstract}

Key words: Soil, particle size distribution, fractals, fragmentation, logselfsimilarity, iterated function system.

\section{Introduction}

Soil PSD is a fundamental soil property that greatly influences soil porosity and mechanical and hydraulic properties. Its description is usually made for soil particles with sizes smaller than $2 \mathrm{~mm}$. A comprehensive description of PSD within this small size interval requires a sophisticated texture analysis, including novel techniques like laser diffraction analysis (see MONTERo and MARTIN, 2003). These analyses have to be repeated for every soil sample, and are highly time-consuming, and expensive.

Attempts to find an equation to simulate the PSD were published in НATCH and Choate (1929), Krumbenn and Pettuohn (1938), Otto (1939), Inman (1952). In Buchan et al. (1993) several equations for the distribution are compared and the authors showed that the best one was a lognormal model.

Dpto. de Matemática Aplicada, E.T.S.I. Agrónomos, Universidad Politécnica de Madrid, 28040 Madrid, Spain. E-mail: carlos.garciagutierrez@upm.es 
Another equation for soil PSD has been derived based on the observed scaling behavior of the number $N(R)$ (or mass) of particles of size greater than a given $R$ : Turcotte, in 1986, showed the scaling rule

$$
N(R) \approx R^{-D},
$$

$D$ being a number called the scaling fractal dimension, which is at present known as Turcotte's Law.

Since then considerable work has been devoted to testing the fractality of the soil PSD (see Anderson et al. 1998, for a review, and Turcotte, 1992; Tyler and WheAtCraft, 1989; Tyler and WheAtCraft, 1992; Wu et al., 1993 for specific results).

Soil PSD is usually reported by providing only mass percentages of clay (particles with sizes $\leq 0.002 \mathrm{~mm})$, silt $(0.002-0.05 \mathrm{~mm})$ and sand $(0.05-2 \mathrm{~mm})$. A method to simulate the distribution from this poor description would be extremely valuable for further use of PSD for various prediction purposes. To attain that objective, a hypothesis on the distribution is needed.

The power scaling (1.1) implies the fractal behavior of the particle size distribution. This fractal behavior of the PSD inspired the use of mathematical self-similar mass distributions to simulate the entire distribution (MARTIN and TAGUAS, 1998). The selfsimilarity hypothesis was further tested in TAGUAS et al. (1999).

In Martin and Garcia-Gutierrez (2006), the model was revised by changing the self-similar hypothesis into a logselfsimilarity assumption about the distribution, based on the fact that the mass of the clay, silt and sand textural fractions is comparable but the size ranges of these fractions $(0.002,0.048$ and $1.95 \mathrm{~mm})$ are only comparable in the log-scale. Random logselfsimilar cascades were used to simulate soil distributions beyond the available data, obtaining a surprising result: The best simulation results are attained when the variance of the random factor is close to 0 , this is, using only exact logselfsimilarity.

The purpose of this paper is to test the strict logselfsimilarity of particle size distributions by means of iterated function systems: We test the capability to simulate the distributions with the minimum number of textural data inputs, that is, three or even only two soil textural fractions. We make a detailed study using a large dataset (USDA-SCS, 1975) and compare the results with those obtained following the previous self-similar hypothesis (MARTIN and TAGUAS, 1998). We also test the characterization of textures via heterogeneity parameters provided by the logselfsimilar model.

The paper is organized as follows: In section 2 the logselfsimilar model is explained in detail. In section 3, we present the materials which are used to test the model and the way in which the model is applied. Section 4 contains the results and section 5 provides the conclusions of this work. 


\section{Theory}

Soil PSD is viewed as a distribution or measure that assigns to any interval $I=[a, b]$ of $\mathbb{R}$, the mass of soil particles whose size (equivalent diameters) is greater or equal to $a$ and less or equal to $b$. Next we present theory related with self-similar mass distributions and the logselfsimilar model.

\subsection{Selfsimilar Mass Distributions (Measures)}

Given a set of functions (linear transformations)

$$
\varphi_{i}: \mathbb{R} \rightarrow \mathbb{R}, \quad\left|\varphi_{i}(x)-\varphi_{i}(y)\right|=r_{i}|x-y|, \quad r_{i}<1, \quad i=1 \ldots m
$$

and a set of positive numbers (probabilities) $p_{i} \geq 0, i=1 \ldots m, \sum_{i=1}^{m} p_{i}=1$, a unique mass distribution $\mu$ exists such that $\mu(\mathbb{R})=1$ and

$$
\mu(J)=\sum p_{i} \mu\left(\varphi_{i}^{-1}(J)\right)
$$

for $J \subset \mathbb{R}$ (HutChinson, 1981).

The set $\left\{\varphi_{i}, p_{i}, i=1 \ldots m\right\}$ is called the iterated function system (IFS) and the mass distribution is said to be the selfsimilar mass distribution of the IFS. The support of the above distribution is the set $I$ which verifies that

$$
I=\bigcup_{i=1}^{m} \varphi_{i}(I) .
$$

Moreover, one has that

$$
\lim _{n \rightarrow \infty} \frac{1}{n+1} \sum_{k=0}^{n} f\left(x_{k}\right)=\int_{\mathbb{R}} f(x) d \mu(x)
$$

for all continuous functions $f: \mathbb{R} \rightarrow \mathbb{R}$, being $x_{k}=\varphi_{k} \circ \cdots \varphi_{2} \circ \varphi_{1}\left(x_{0}\right)$, for all $x_{0} \in \mathbb{R}$. In particular this implies that, if $I \subset \mathbb{R}$ is an interval and $m(n)$ is the number of points of $\left\{x_{0}, \ldots, x_{k}\right\} \cap I$, then (see MARTIN and TAguas, 1998)

$$
\mu(I)=\lim _{n \rightarrow \infty} \frac{m(n)}{n+1} .
$$

Self-similar measures are common examples of so-called multifractal measures, that is, measures for which the local Hölder exponent of $\mu$ at $x$ defined by the limit

$$
\alpha(x)=\lim _{r \rightarrow 0} \frac{\log \mu\left(I_{r}(x)\right)}{\log r}
$$

is not constant on the support, $I_{r}(x)$ being the real interval $[x-r, x+r]$.

The Hölder exponents for self-similar measures typically span the entire interval between two extreme values $\alpha_{\min }$ and $\alpha_{\max }$ (see Everstz and MANDELBRot, 1992 or 
FALCONER, 1997, for further details). The exponent provides a measure of mass concentration around the point: The greater $\alpha(x)$ is, the smaller will be the mass concentration and vice versa. However, for simulated self-similar measures (or experimental measures with self-similar characteristics), the above theoretical approach is replaced with a coarse version involving a scaling analysis of overall information quantities instead of the pointwise local Hölder exponents lacking practical sense in a natural setting. One common choice is to consider dyadic scaling down (EVERSTz and MANDELBRot, 1992), that is, succesive partitions of $I$ of size $L \cdot \varepsilon=L \cdot 2^{-k}, L$ being the length of $I$ and $k=1,2,3, \ldots$ At every size scale $\varepsilon$, a number $N(\varepsilon)=2^{k}$ of subintervals (cells) $I_{i}, i=1 \ldots N(\varepsilon)$ are considered and their respective measures $\mu\left(I_{i}\right)=\mu_{i}(\varepsilon)$ assumed to be provided by available data. Now, the ratio $\log \mu_{i}(\varepsilon) / \log \varepsilon$ is called the coarse Hölder exponent of interval $I_{i}$ and the Holder spectrum is defined via a parameter $q$ such that

$$
\alpha(q) \approx \frac{\sum_{i=1}^{N(\epsilon)} \mu_{i}(q, \epsilon) \log \mu_{i}(\epsilon)}{\log \epsilon},
$$

where

$$
\mu_{i}(q, \epsilon)=\frac{\mu_{i}(\epsilon)^{q}}{\sum_{i=1}^{N(\epsilon)} \mu_{i}(\epsilon)^{q}}
$$

and " $\approx "$ means that a suitable linear fitting holds for a range of scales ( $\varepsilon$ values) where we want to characterize the scaling regularity of the measure $\mu$ (see EvERSTz and Mandelbrot, 1992; ChHABra and Jensen, 1989 for details).

For exact self-similar measures, " $\approx$ " in equation (2.6) may be replaced with the limit when $\epsilon \rightarrow 0$. In such a case, the function $\alpha(q)$ for $-\infty<q<+\infty$ parameterizes the interval $\left[\alpha_{\min }, \alpha_{\max }\right]$ of local Hölder exponents.

On the other hand, a suitable fitting of equation (2.6) applied to experimental data, for a certain range of scales, may reveal that the measure concerned has self-similar features within that range.

\subsection{The Logselfsimilar Model}

Soil PSD is defined by assigning to each interval $I=[a, b] \subset \mathbb{R}$ the mass $\mu(I)$ of particles whose size is in that interval. This distribution can be seen as the result of a fragmentation process, this is, an iterative processs acting within a range of scales. Experimental data on this distribution showed power scaling of the type reflected in Section 1 (AnDERSON et al., 1998). This fact suggests that the distribution should have scale-invariant behavior: If we zoom into the mass interval to see it at a finer scale, it should resemble (statistically) the structure of the whole interval: A photograph of soil looks similar at every scale; it is impossible to guess the size of elements in the picture.

Once the invariance with respect to the scale becomes a sensible hypothesis, the problem that arises is to determine how this invariance-based model can be used. MARTín 
and TAGUAS (1998) proposed a self-similar model generated by iterated function systems (IFS) that was useful for simulating self-similar PSDs from the knowledge of common textural data (clay, silt and sand mass proportions). This model generates a self-similar mass distribution via an iterative process that allocates the relative mass proportions of the elementary size classes in reduced linear copies of the size interval. Testing this model showed that the use of the clay (soil particles smaller than $0.002 \mathrm{~mm}$.), silt (2-50 $\mathrm{mm})$ and sand $(50-2000 \mathrm{~mm})$ fractions, for example, as inputs for the model lead to a very unrealistic simulated PSD. The reason is that these three fractions (subintervals of the mass size distribution) contain similar amounts of mass, however the respective sizes of the intervals differ by orders of magnitude (viz. $0.002 \mathrm{~mm}, 0.048 \mathrm{~mm}$ and $1.95 \mathrm{~mm}$, respectively). The simulations lead to vast amounts of soil mass accumulated in very small linear copies of the size interval (specifically in the reduced linear copies of the clay interval), which contradicts common pedological knowledge.

Montero and Martin (2003) computed the Hölder spectrum of soil texture data obtained with laser diffraction, and the scaling behavior was excellent when the interval of sizes was log-rescaled. In fact, using the log-rescaled interval instead of the usual interval in scaling analysis is strongly supported by the nature of the data provided by texture analysis instruments (see Martín et al., 2001; Montero and MARTín, 2003).

These facts suggested a reconsideration of the selfsimilar model (MARTIN and GARCIAGutierrez, 2006). The key idea is to view the PSD as the result of an iterative process that spreads the mass in the log-rescaled particle size interval. Such property is called logselfsimilarity. In MARTIN and GARCIA-GutierREZ (2006) random cascades were used to simulate the PSD. The random factor of the model agglomerates all the causes different to logselfsimilarity that could explain the PSD. When variance is 0 , the random factor disappears, and logselfsimilarity is the only explanation for the soil PSD. The best simulation results were attained when the random factor was 0 , therefore supporting the logselfsimilar behavior of the distribution. This fact lead us to using strict logselfsimilarity and simulating the PSD by utilizing the iterated function system (IFS).

Below we describe an IFS simulation for a PSD based on the logselfsimilarity hypothesis. It is a simple algorithm by which we can obtain the mass of soil particles with sizes within a given interval $I \subset I_{0}$, being $I_{0}=[0,2000]$ the textural interval.

Let $p_{i}$ be the mass proportions of soil particles corresponding to the size fractions $I_{i}$, $i=1 \ldots q$. Thus $I_{0}=\cup_{i=1}^{q} I_{i}$.

Let $\xi_{i}$ be the linear transformations that map $I_{0}$ into $I_{i}$. That is, if $I_{i}=[a, b]$, then $\xi_{i}(x)=x(b-a) / 2000+a$. Also let $I_{i}^{*}=\Phi\left(I_{i}\right)=[\log (1+a), \log (1+b)]$. The new linear transformations, $\varphi_{i}$, are the ones that transform $I_{0}^{*}$ into $I_{i}^{*}$, with the same probabilities $p_{i}, i=1,2,3$.

Then begins the iteration procedure:

(1) Take any starting point $x_{0}$ from the support $I_{0}^{*}$.

(2) Choose randomly, with probability $p_{i}$, one of the three linear transformations $\varphi_{i}$, $i=1,2,3$ and calculate the next point of the simulation: $x_{1}=\varphi_{i}\left(x_{0}\right)$. 
(3) Continue the process as in (2), obtaining all the points of the simulation: $x_{k}=\varphi_{i}\left(x_{k-1}\right)$, with probability $p_{i}$, chosen randomly, $i=1,2,3$.

This process defines a limit measure that is multifractal.

With the points $x_{0}, x_{1}, \ldots, x_{n}$ we can obtain the measure at any interval $I \subset I_{0}, \mu(I)$, by

$$
\mu(I)=\lim _{n \rightarrow \infty} \frac{m(n)}{n+1}
$$

$m(n)$ being the number of points of the orbit $x_{i}$ that fall within the interval $I$.

For this we have to calculate $I^{*}=\Phi(I)$ and count $m^{*}(n)$, the number of points of the orbit that fall within $I^{*}=\Phi(I) \subset I_{0}^{*}$. Then we calculate $\mu\left(I^{*}\right)=\mu(I)$.

The estimate of $\mu(I)$ is obtained very quickly in the practice, since the convergence of the algorithm is extremely rapid. In fact, computed $\mu(I)$ did not change after $n=3000$.

\subsection{Heterogeneity Parameters}

Soil PSD is used in most pedotransfer functions, that is, functions that estimate certain soil properties that are difficult or expensive to measure, in an indirect way via empiric correlations (VAN Genuchten and Leu, 1992; Wösten et al., 2001). For example, soil hydraulic properties are estimated by using parameters that characterize the shape of the PSD. Therefore PSD characterization and subsequently soil textral classification is an important issue in soil sciences.

The USDA textural triangle is the most common way to classify soil textures. It uses the standard PSD available data (clay, silt and sand mass fractions) to classify the soils in 13 different types (textural classes), according to specific mass fraction boundaries for each class. Other classification systems (FoLK, 1954; SHEPARD, 1954; BAvER et al., 1972; VANONI, 1980) follow the above scheme, only with variations of the mass fraction boundaries, however these classifications are rather poor because soils with very different physical properties may fall under the same class.

Also, the use of previously mentioned fractal dimensions in soil classification proved useless, as texturally different soils can have the same dimension. There is a need to develop additional parameters to characterize soil structure that might be better predictors of soil properties.

The entropy dimension is a parameter that measures and characterizes the degree of heterogeneity of a complex distribution. Is it difficult to obtain this parameter directly from a distribution, but a well-known result from fractal geometry (YounG, 1982; DELIU et al., 1991) allows one to compute this parameter with a simple formula when the distribution is (or is assumed to be) self-similar. This assumption, earlier used to describe and simulate the PSD, was later used in MARTIN et al. (2001) to parameterize the soil texture with entropy dimensions. The textural triangle regions were changed for intervals of values of entropy dimensions, thus obtaining a continuous parameterization of soil texture. 
MARTIN et al. (2005) used the balanced entropy, which corrects the distortion of the entropy when the size of the intervals are not equal, to parameterize soil texture and predict soil volumetric water content.

Hölder exponents provide information about the mass of particles with sizes within various ranges, and can be related to physical or hydraulic properties related to the packing of particles, like soil water retention. These heterogeneity parameters can be obtained from available texture data, using the model's logselfsimilarity hypothesis.

The Hölder spectrum $\alpha(q)$ of the measure $v$ on the rescaled interval is given by (see FALCONER, 1994, 1997)

$$
\alpha(q)=\frac{\sum_{i=1}^{m} p_{i}^{q} r_{i}^{\beta} \log p_{i}}{\sum_{i=1}^{m} p_{i}^{q} r_{i}^{\beta} \log r_{i}},
$$

where $\beta=\beta(q)$ is a positive number verifying

$$
\sum_{i=1}^{m} p_{i}^{q} r_{i}^{\beta(q)}=1 .
$$

The value

$$
\alpha(0)=\frac{\sum_{i} r_{i}^{*} \log p_{i}}{\sum_{i} p_{i} \log r_{i}^{*}}
$$

would approach the average value of the coarse Hölder exponents for fine partitions of the size interval and

$$
\alpha(1)=\frac{\sum_{i} p_{i} \log p_{i}}{\sum_{i} p_{i} \log r_{i}^{*}}
$$

is consistent with the entropy dimension of the distribution, $v$, mentioned above.

These heterogeneity parameters, obtained through the new logselfsimilarity hypothesis (MARTin and Garcia-Gutierrez, 2006) can be used to characterize the PSD heterogeneity, to quantitatively classify the soils, and potentially to estimate soil hydraulic properties.

\section{Materials and Methods}

The data used to simulate and test the logselfsimilar theory corresponded to the upper two horizons of soils reported by the Soil Conservation Service (1975). Soil data included the mass proportions $m_{i}$ of particles in eight size classes $(\mathrm{mm})$ : clay $(<0.002)$, silt $(0.002-0.02)$ and $(0.02-0.05)$, very fine sand $(0.05-0.1)$, fine sand $(0.1-0.25)$, medium sand $(0.25-0.5)$, coarse sand $(0.5-1)$ and very coarse sand (1-2). In order to use these data to construct an IFS, we shall denote by $[a, b]$ the particles with sizes greater than or equal 
to $a$ and less or equal to $b$. These size classes determine a set of seven intermediate cutoff points $0.002,0.02,0.05,0.1,0.25,0.5,1$ and eight consecutive intervals corresponding to the eight size classes $I_{1}=[0,0.002], I_{2}=[0.002,0.02], \ldots, I_{8}=[1,2]$.

These data offer the possibility of using some of them as inputs for the model and simulate a fractal soil PSD associated with it. The simulated data then can be compared with the real data, not used as input for the model to estimate the goodness-of-fit of the simulation.

We also try to find which intervals, from all available in one soil, used as input values for the model, yield the best simulation results.

The number of linear transformations in the simulation vary from 2 to 8 , according to the available data. With 2 linear transformations the number of cutoff points is 7 , thus there are 7 different input value possibilities. With 3 linear transformations the number of input value possibilities is 21 ; with 4 linear transformations it is 35 . Thus, the method provides a great number of potential simulated PSDs.

To run most of the tests on the model we used 3 linear transformations. The reason for this choice is that three is the number of the most commonly available textural data, namely: the mass percentages of clay, silt and sand. In some cases additional simulations were made following TAGUAS et al. (1999), in order to compare those results to the ones obtained with the logselfsimilar cascade model. Both simulation methods use the same soil data as inputs and for comparison with their simulated counterparts.

The model was also tested with two linear transformations and the results were compared with the use of three linear transformations.

We constructed the three linear transformations with different possible logselfsimilar IFS

$$
\left\{\varphi_{1}, \varphi_{2}, \varphi_{3} ; p_{1}, p_{2}, p_{3}\right\}
$$

by using the following procedure:

(1) Select two cutoff points $\alpha$ and $\beta$ among the seven possible choices, $\alpha<\beta$.

(2) Let $p_{1}, p_{2}$ and $p_{3}$ be the mass proportions of the three constructed intervals $I_{1}=[0, \alpha]$, $I_{2}=[\alpha, \beta]$ and $I_{3}=[\beta, 2000]$.

(3) Obtain the log-rescaled intervals $I_{i}^{*}=\Phi\left(I_{i}\right)$, and assign them the same probability as to the initial intervals. Also calculate the set $I^{*}=\Phi(I)$

(4) Let $\varphi_{i}$ be the linear transformation which maps the interval $I^{*}$ into $I_{i}^{*}$.

These rules permit us to make up to 21 IFS simulations for each soil, depending on the values of the two cutoff points $(\alpha$ and $\beta$ ). Another 21 simulations were performed in accordance with the self-similar scheme of TAGUAS et al. (1999). The results obtained with these methods were compared via the error of the respective simulations, defined as

$$
\epsilon=\frac{\sum\left|m_{i}-m_{i}^{\prime}\right|}{2}
$$


$m_{i}$ being the real mass proportion in the size class $I_{i}$, and $m_{i}$ the mass proportion assigned to the same size class by the simulation. This error was used in TAGUAs et al. (1999), where the self-similar model was tested, therefore we use the same formula in order to keep coherence on the testing method. A similar formula was employed earlier in VRSCAY (1991). The rationale for dividing by two 3.1 in is the following: the formula adds mass deviations for the intervals. A positive mass deviation in one interval derives from a negative one in another, and vice versa. In some way, mass deviations are accounted two times, therefore we divide by two.

For each soil 21 different simulations were created with the different input value possibilities, and 21 different error values were obtained. We used the minimum one as the error of the PSD simulation because it corresponds to the best simulation result with the logselfsimilar model (using only three data).

The above scheme varies slightly when using 2 linear transformations (instead of 3 ). In this case there is only one possible cutoff point choice, and the number of intervals is only 2 . The number of possible IFSs per soil is 7 when the first interval starts at zero, but we also tested the IFS when the first interval starts at 0.002 , this is ignoring the clay fraction. The number of possible IFs in this case is 6 , which is the number of possible cutoff points $\{0.05,0.1,0.25,0.5,1\}$.

The error in this case was also calculated with 3.1 .

\section{Results and Discussion}

Textural data of 171 soils have been studied. We excluded soils whose mass proportions were polarized into any of the three classes (silt, clay or sand). Therefore, the soils whose clay and sand content was more than $85 \%$ and those whose silt content was more than $90 \%$ were eliminated from the list. The number of remaining soils was 158 .

First we used three linear transformations ( 3 subintervals or mass proportions) as input values for the model to compare it to the previous self-similar model (TAGUAs et al., 1999). For 111 soils (70.3\% of the selected soils) the error was smaller in the logselfsimilar model than in the self-similar.

The average error of the logselfsimilar simulations was 10.9 whereas the average error for the self-similar ones was 14.7 .

With 3 linear transformations the error for each soil is the minimum of the errors of the simulations with the 21 different input value possibilities. For the self-similar case the minimum is attained with the 0-0.002-0.020-2 partition as input values on 90 out of the 158 soils $(57 \%)$. The next partition with the most minimums is $0-0.05-0.1-2$. For the logselfsimilar case the minimum is attained with the partition $0-0.002-0.02-2$ in 34 soils $(21.5 \%)$ and with the partition $0-0.002-0.5-2$ in the other 22 soils $(13.9 \%)$.

Another test was to measure the error on all the soils for the clay, silt and sand fractions, which are the most readily available soil data, in the logselfsimilar model 
and in the self-similar model. Clay particles have diameters between 0 and $0.002 \mathrm{~mm}$, silt particles have diameters between 0.002 and $0.02 \mathrm{~mm}$ in the ISSS classification, and between 0.002 and $0.05 \mathrm{~mm}$ in the USDA classification. The particles with sizes between the upper limit of the silt and $2 \mathrm{~mm}$ are considered to be sand particles. Therefore the two input values tested were 0-0.002-0.02-2 (ISSS) and 0-0.002-0.05-2 (USDA). In the ISSS partition case the mean error was 20.0 for both cases. In 93 soils (58.9\%) the error was smaller in the logselfsimilar simulation. For the USDA partition the mean error was 29.6 for the self-similar case and 23.9 for the logselfsimilar case. In 127 soils $(80.3 \%)$ the error was smaller in the logselfsimilar simulation of the soil PSD.

By using 2 initial linear transformations (two input values), instead of 3, we compared the results with the self-similar model using the same number of linear transformations. This test was done in two ways: with the first interval starting from size 0 and with the first interval starting from size 0.002 , this is, not counting the clay subinterval. The mean value of the error of all the soils tested (158, the selection mentioned above) was 22.3 for the logselfsimilar model and 30.3 for the selfsimilar one, when the first interval contained the clay fraction. When the clay fraction was not included in the simulation, this is, when the first interval started at $0.002 \mathrm{~mm}$., the mean value of the error was 37.7 for the logselfsimilar case and 41.2 for the selfsimilar case.

The results demonstrate that the logselfsimilarity hypothesis is more realistic than the previous self-similar hypothesis when using only two linear transformations. The results also show that the use of two linear transformations yields greater errors than when using 3 linear transformations. When increasing the number of inputs in the model, soil PSDs are simulated better. The number of input values depends on the textural fractions that are known from the soil, which is usually three. The results also show that the soil particles in the clay fraction are also involved in the fractal logselfsimilar structure of the PSD. This is a surprising and not expected result because clay particles are presumably formed not as a result of a fragmentation process, which is the explanation for the fractal logselfsimilar structure of the PSD.

The Hölder spectrum $\alpha(q)$ for all soils was calculated for several values of $q$. Table 1 shows the mean values of $\alpha(0), \alpha(1)$ and $\alpha(1) / \alpha(0)$ for the different textural classes of the USDA textural triangle in all 171 soils. For this parameter the Loam, Sandy Loam and Sandy Clay Loam classes are not distinguishable. The same is true for the Silt Loam and Silty Clay Loam classes. Nonetheless, when varying the parameter $q$ the Hölder spectrum is capable of distinguishing between those classes: Table 2 shows the Hölder parameters for the previous classes with $q=2,-2,10,-10$.

The heterogeneity parameter wellness depends on the chosen model. The better the model, the more accurately the parameters will quantitatively characterize the texture and, in addition, the better to establish regressions with soil physical properties or build new pedotransfer functions. 
Table 1

Mean values of $\alpha(0), \alpha(1)$ and $\alpha(1) / \alpha(0)$ for the different textural classes

\begin{tabular}{llll}
\hline Textural Class & \multicolumn{1}{c}{$\alpha(0)$} & $\alpha(1)$ & $\alpha(1) / \alpha(0)$ \\
\hline Sand & 1.65954067 & 0.624458 & 0.37994797 \\
Loamy Sand & 1.3023216 & 0.7883644 & 0.61170227 \\
Sandy Loam & 1.14006598 & 0.89193242 & 0.9493417 \\
Silt Loam & 1.37489666 & 0.77902314 & 0.59723171 \\
Silt & 1.56655933 & 0.646987 & 0.4217506 \\
Loam & 1.14928982 & 0.90012724 & 0.79933527 \\
Sandy Clay Loam & 1.137707 & 0.8940058 & 0.79315775 \\
Clay Loam & 1.19510946 & 0.87178831 & 0.73544949 \\
Silty Clay Loam & 1.3627452 & 0.77143413 & 0.58778276 \\
Silty Clay & 1.43659986 & 0.71459671 & 0.52387275 \\
Clay & 1.42989975 & 0.60704275 & 0.4406898 \\
\hline
\end{tabular}

Table 2

Mean values of $\alpha(2), \alpha(-2), \alpha(10)$ and $\alpha(-10)$ for some textural classes

\begin{tabular}{lllll}
\hline Textural Class & \multicolumn{1}{c}{$\alpha(2)$} & $\alpha(-2)$ & $\alpha(10)$ & $\alpha(-10)$ \\
\hline Sandy Loam & 0.7787254 & 1.68533752 & 0.648082 & 1.82205762 \\
Silt Loam & 0.67265007 & 2.22231493 & 0.56712528 & 2.33494603 \\
Loam & 0.80783118 & 1.69722282 & 0.68345041 & 1.81338135 \\
Sandy Clay Loam & 0.785247 & 1.678321 & 0.6553278 & 1.7969594 \\
Silty Clay Loam & 0.65487927 & 2.03840833 & 0.56835773 & 2.15139607 \\
\hline
\end{tabular}

\section{Conclusions}

The fractal logselfsimilar hypothesis for the structure of the soil PSD was tested in this work. The new model was compared to the previous self-similar one on quality data and was found to substantially improve the simulation of the soil PSD.

When using 3 input values the errors of the logselfsimilar simulations were smaller than the errors when using the self-similar model for $70 \%$ of soils and the average error was 10.9 , in contrast to 14.7 , which was the average error with the previous model. The error mean values when using just two input values were 22.3 and 30.6 , respectively.

The error of the simulations depended greatly on the input values used. The best input values for the logselfsimilar model are the mass of particles with sizes in the following intervals $[0,0.002],[0.002,0.02]$ and $[0.02,2]$, which correspond to the clay, silt and sand fractions under the ISSS classification.

The heterogeneity parameters provided by the model can quantitatively characterize soil texture and may be used to build new pedotransfer function or be related to soil physical properties related to PSD. 


\section{Acknowledgements}

This work has partially been supported by Plan Nacional de Investigación Científica, Desarrollo e Innovación Tecnológica (I+D+I) under ref. AGL2007-62648 Spain and by DGUI (Comunidad de Madrid) - UPM (Technical University of Madrid), Ref.:M0700204135. We thank the reviewers for their valuable comments and suggested changes. They significantly helped us to enhance the paper.

\section{REFERENCES}

Anderson, A.N., McBratney, A.B., and Crawford, J.W. (1998), Applications of fractals to soil studies, Adv. Agron. 63, 1-76.

BARNSLEY, M.F. and DemKo, S. (1985), Iterated function systems and the global construction of fractals, Proc. R. Soc. Lond. A 399, 243-275.

Barnsley, M.F., Erwin, V., Hardin, D., and Lancaster, J. (1985), Solution of an inverse problem for fractals and other sets, Proc. Natl. Acad. Sci. U.S.A. 83, 1975-1977.

BaVter, L.D., Gardner W.H., and GardNer, W.R. Soil physics (4th edn. Wiley, NY 1972).

Buchan, G.D., Grewal, K.S., and Robson, A.B. (1993), Improved models of particle-size distribution: An illustration of model comparison techniques, Soil. Sci. Soc. Am. J. 57, 901-908.

ChHabra, A. and Jensen, R. V. (1989), Direct determination of the $f(\alpha)$ singularity spectrum, Phys. Rev. Lett. 62, $1327-1330$.

Deliu, A., Geronimo, J.S., Shonkwlller, R., and Hardin, D. (1991), Dimensions associated with recurrent selfsimilar sets, Math. Proc. Camb. Phil. Soc. 110, 327-336.

Everstz, C.J.G and Mandelbrot, B.B. Multifractal measures In Chaos and Fractals (eds. Peitgen, H., Jürgens H., and Saupe D.) (Springer, Berlin, 1992) pp. 921-953.

FALCONER, K.J. (1994), The multifractal spectrum of statistically self-similar measures, J. Theoret. Prob. 7 (3), $681-702$.

Fatconer, K.J. Techniques in Fractal Geometry (Wiley \& Sons, Chichester 1997).

FolK, R.L. (1954), The distinction between grain size and mineral composition in sedimentary rock nomenclature, J. Geol. 62, 344-359.

НАтсн, T. and ChOATE, S. (1929), Statistical description of the size properties of nonuniform particulate substances, J. Franklin Inst. 207, 369-387.

Hutchinson, J. (1981), Fractals and selfsimilarity, Indiana Univ. J. of Mathematics 30, 713-747.

InMAN, D.L. (1952), Measures for describing the size distribution of sediments, J. Sediment. Petrol. 22 (3), $125-145$.

Krumbein, W.C. and Pettijohn, F.J. Manual of Sedimentary Petrography (Appleton-Century, New-York 1938).

Mandelbrot, B.B. The Fractal Geometry of Nature (New York, W.H. Freeman, 1982).

Martin, M.A. and Taguas, F.J. (1998), Fractal modelling, characterization and simulation of particle-size distributions in soil, Proc. R. Soc. Lond. A 454, 1457-1468.

MARTIN, M.A., REY, J.M., and TAGUAS, F.J. (2001), An entropy-based parameterization of soil texture via fractal modelling of particle-size distribution, Proc. R. Soc. Lond. A 457, 937-947.

Martin M.A., Pachepsky, Y.A., Rey, J.M., Taguas, J., and Rawls, W.J. (2005), Balanced entropy index to characterize soil texture for soil water retention estimation, Soil Sci. $170,(10)$.

MARTIN, M.A. and GARCLA-GUTIERREZ, C. (2008), Log selfsimilarity of continuous soil particle-size distributions estimated using random multiplicative cascades, Clays and Clay Minerals, 56 (3), 389-395.

MonTero, E. and MaRTin, M.A. (2003), Hólder spectrum of dry grain volume-size distributions in soil, Geoderma 112, 197-204.

Отто, G. (1939), A modified logarithmic probability graph for interpretation of mechanical analysis of sediments, J. Sediment. Petrol. 9 (2), 62-76.

ShEPARD, F.P. (1954), Nomenclature based on sand-silt-clay ratios, J. Sediment. Petrol. 24, 151-158. 
Shmazi, M.A. and BoErsma, L. (1984), A unifying quantitative analysis of soil texture: Improvement of precision end extension of scale, Soil. Sci. Soc. Am. J. 52, 181-190.

Taguas, F.J., Martin, M.A., and Perfect, E. (1999), Simulation and testing of self-similar structures for soil particle-size distributions using iterated function systems, Geoderma. 88, 191-203.

TurcotTe, D.L. (1886), Fractals and Fragmentation, J. Geophys. Res. 91, 1921-1926.

Turcotte, D.L., Fractals and Chaos in Geology and Geophysics (Cambridge University Press, Cambridge 1992).

TYler, S.W. and WHEATCRAFT, S.W. (1989), Application of fractal mathematics to soil water retention estimation, Soil Sci. Soc. Am. J. 53, 987-996.

Tyler, S.W. and Wheatcraft, S.W. (1992), Fractal scaling of soil particle-size distributions: analysis and limitations, Soil Sci. Soc. Am. J. 56, 362-369.

Young (1982)

VAN GENUChTEN, M.T.H. and LeIJ, F., On estimating the hydraulic properties of unsaturated soils. In Proc. Int. Workshop on Indirect Methods for Estimating the Hydraulic Properties of Unsaturated Soils (eds. van Genuchten, M.T.H., Leij, F.J., and Lund, L.J.) (University of California, Riverside, CA 1992).

VANon, V.A., Sedimentation Engineering (Sedimentation Committe, Hydraulic Division, American Society of Civil Engineers, New York 1980).

VRSCAY, E.R., Moment and collage methods for the inverse problem of fractal construction with iterated function systems. In Fractals in the Fundamental and Applied Sciences (eds. Peitgen, H.O., Henriques, J.M., and Penedo, J.L.) (North Holland 1991) pp. 443-461.

Wösten, J.H.M., PachePsky, J.A., and Rawls, J.W. (2001), Pedotransfer functions: Bridging the gap between available basic soil data and missing soil hydraulic characteristics, J. Hydrol. 251, 123-150.

Wu, Q., Borkovec, M., and STICHER, H. (1993), On particle size distributions in soils, Soil Sci. Soc. Am. J. 57 , 883-890.

Young, L.S. (1982), Dimension, entropy and Liapunov exponents, Ergod. Th. Dynam. Sys. 2, 109-124. 